\title{
An Improved Intragastric Balloon Procedure Using a New Balloon: Preliminary Analysis of Safety and Efficiency
}

\author{
Gustavo L. Carvalho • Cesar B. Barros • \\ Masaichi Okazaki • Moacir L. Novaes • \\ Pedro C. Albuquerque • Nair C. Almeida • \\ Pedro Paulo C. Albuquerque • Chika Wakiyama • \\ Thiago G. Vilaça • José Sergio N. Silva • \\ Rapahel M. Coelho
}

Received: 28 April 2008 / Accepted: 23 May 2008 / Published online: 26 June 2008

(C) The Author(s) 2008

\begin{abstract}
Background The authors developed a new intragastric balloon procedure with the objective of making it safer, faster, and less expensive than the established ones. The proposed procedure uses a new gastric balloon with technical improvements in the placement and removal procedures.

Methods From June 2006 to July 2007, 52 patients were submitted to the new treatment with the Silimed Gastric Balloon (SGB), as part of a multidisciplinary program involving clinical, psychological, and behavioral approaches.

Results The new placement and removal procedures of the SGB were effective and safe in all the cases. Due to simplicity and shortened duration of the procedures, all the patients left the outpatient clinic in less than $1 \mathrm{~h}$ after the placement or removal of the SGB. For the 14 patients who had completed the 6-month treatment, the initial mean weight, mean body mass index (BMI), and mean excess of weight (EW) were, respectively, $100.7 \mathrm{~kg}, 35.7 \mathrm{~kg} / \mathrm{m}^{2}$, and
\end{abstract}

G. L. Carvalho $(\bowtie) \cdot$ M. Okazaki $\cdot$ M. L. Novaes $\cdot$

P. C. Albuquerque $\cdot$ N. C. Almeida P. P. C. Albuquerque $\cdot$

C. Wakiyama $\cdot$ T. G. Vilaça J. S. N. Silva $\cdot$ R. M. Coelho

School of Medicine (FCM), University of Pernambuco (UPE),

Recife, Pernambuco, Brazil

e-mail: gc@elogica.com.br

\section{B. Barros}

Clinical Research Department,

Silimed Product Support Department,

Rio de Janeiro, Rio de Janeiro, Brazil
$30.0 \mathrm{~kg}$. After the 6-month treatment, these values decreased significantly: $89.4 \mathrm{~kg}, 31.8 \mathrm{~kg} / \mathrm{m}^{2}$, and $19.6 \mathrm{~kg}$. Conclusions Preliminary data suggest that the procedure with the new balloon comes forth as a safe and effective alternative to the treatment of weight loss in patients with appropriate indication of use.

Keywords Gastric balloon - Endoscopic treatment . Endoscopic procedures · Safety $\cdot$ Efficiency $\cdot$ BMI $\cdot$ EWL . Weight loss $\cdot$ Massive obesity

\section{Introduction}

In the last few years, obesity has become one of the main worrisome public health problems of epidemic proportions, both in developed and developing countries. This motivated the specialists in bariatric medicine to continually improve the established therapies for obesity treatment and develop new procedures that can effectively address aspects, such as safety and efficiency.

Among the recently improved minimally invasive procedures, intragastric balloon has been one temporary nonsurgical option that can promote weight loss in select groups of obese patients by partially filling their stomach and inducing a sense of early satiety [1-4].

In the present study, the authors propose a series of technical improvements in the intragastric balloon procedure with the objective of making it safer, faster, and less expensive than the established ones. The proposed procedure uses a new device - Silimed Gastric Balloon (SGB) with technical improvements in the placement and removal procedures. Preliminary results of safety and efficiency of 
the placement and removal procedures are presented and discussed along with preliminary data on weight loss and adverse effects in the patients who had completed the 6month treatment.

\section{Methods}

\section{Patients}

This study included 52 patients ( 45 women), with mean age of $37.1 \pm 10.5$ years (15 to 65 years). They comprised two groups of preobese and obese patients who failed to respond to previous clinical treatment for weight loss: those who did not meet the IFSO standards for bariatric surgery, and those who were not willing to undergo bariatric surgery. Their pretreatment mean weight was $96.5 \pm 22.5 \mathrm{~kg}$ (58.3 to $157 \mathrm{~kg}$ ), mean BMI was $34.7 \pm$ $5.2 \mathrm{~kg} / \mathrm{m}^{2}$ (25.6 to $\left.50.1 \mathrm{~kg} / \mathrm{m}^{2}\right)$, and mean EW was $27.6 \pm$ $16.6 \mathrm{~kg}$ (1.6 to $78.7 \mathrm{~kg})$.

\section{Device}

The SGB design specifications are based on some requirements defined in the 1987 Tarpon Springs's International Workshop, for the safety and efficiency of intragastric balloon designs [5].

The SGB is supplied empty, and is delicately rolled up inside a thin silicon sheath. This makes its placement and positioning in the gastric fundus possible by endoscopic route. The device consists of a smooth and transparent silicon shell that acquires a round format when filled with saline solution. The filling is done by a tube with a polytetrafluorethylene needle at its extremity, which is connected to a self-sealing valve attached to the device shell.

\section{Initial Protocol}

The preprocedure was conducted by a multidisciplinary team that comprised psychologist, nutritionist, bariatric surgeon, and endoscopist. At this stage, information regarding the patients' eating habits and their previous obesity treatments was compiled.

For the SGB procedure, the authors considered the following as absolute contraindications: the presence of hiatal hernia $>5 \mathrm{~cm}$, active peptic ulcer, severe esophagitis (III and IV degree-Savarry Miller), hemorrhagic risk (e.g., esophagic or gastric varicose veins, hemorrhagic gastritis), Crohn's disease, cancer, diverticule and/or esophagic stenosis, serious cardiopulmonary, renal or hepatic disease, previous gastric surgery, psychological disturbances, sweeteaters, and lack of motivation or reluctance to follow the treatment protocol. Free and informed consents from the patients were obtained only after explaining to them the need of the behavioral changes after the SGB placement, the importance of the follow-up visits, and the risks inherent to this procedure.

The SGB placement procedure was immediately preceded by a diagnostic esophagogastroduodenoscopy to define the gastric anatomy, verify the existence of structural abnormality, if any, in the clinical conditions that are contraindicative to SGB placement, and aspirate the gastric content when necessary. After removing the endoscope, the SGB was lubricated with surgical lidocaine gel to initiate the insertion procedure.

\section{Placement and Removal Procedures}

Both procedures were performed under the usual sedation of diagnostic endoscopy. In the placement procedure, the extremity of SGB's sheath was carefully anchored to the endoscope extremity by using a polipectomy snare (Fig. 1).
Fig. 1 a The polipectomy snare carefully tying the extremity of the SGB's sheath (only the extremity of the sheath, and not of the shell, is tied so that the shell does not get damaged); b Anchoring of the extremity of the SGB's sheath to the extremity of the endoscope
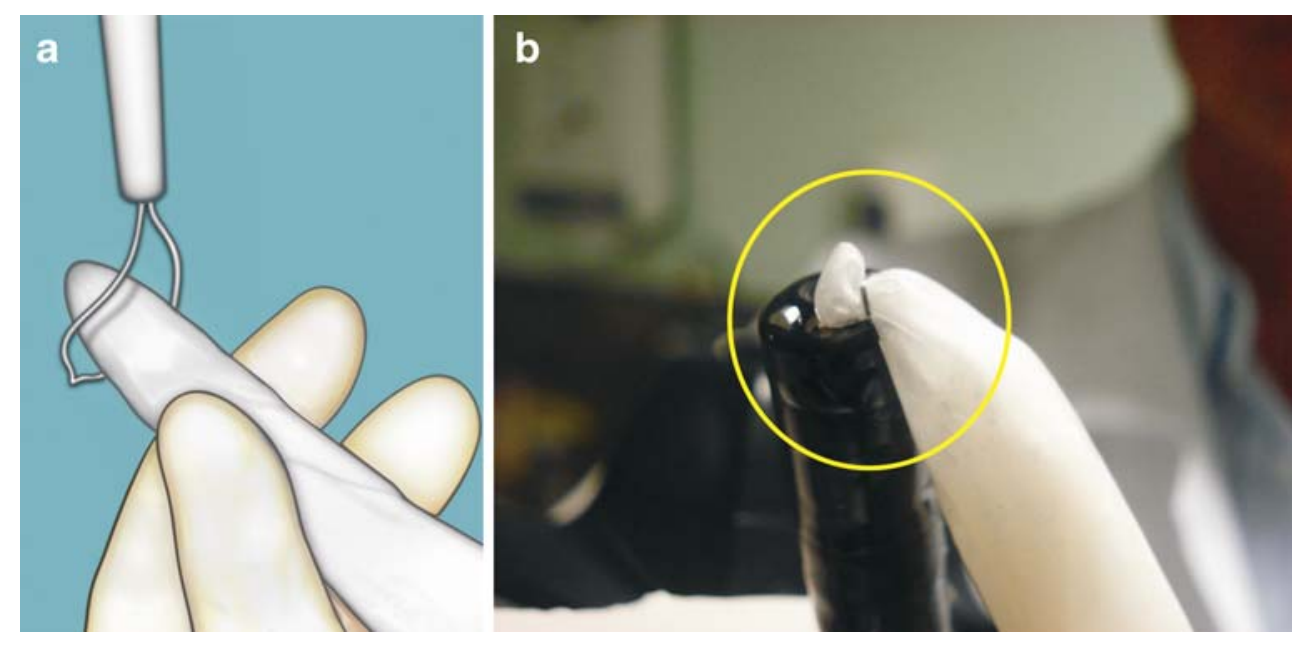
Fig. 2 To position the SGB in the stomach, instead of pushing the SGB without visual examination, as in an orogastric probe, it is pulled by traction of the endoscope under visual examination
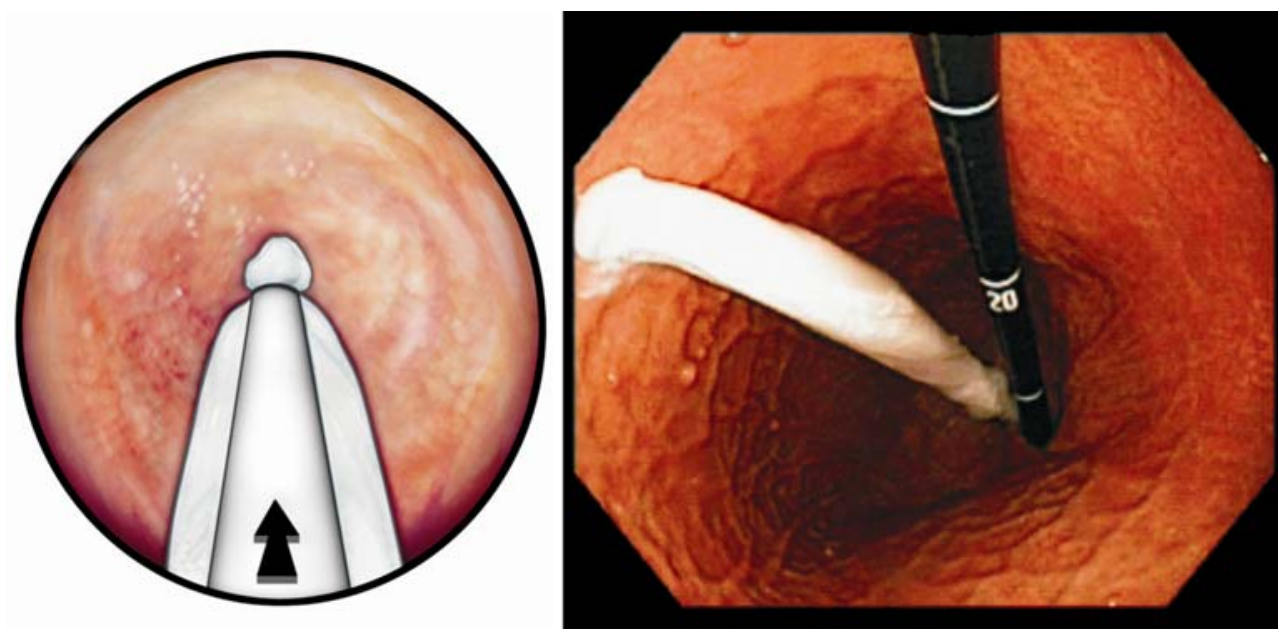

Then, the SGB was smoothly inserted into the stomach by traction under direct visual examination (Fig. 2), released by the polypectomy snare near the pylorus and finally positioned in the gastric fundus by retrocession maneuver followed by SGB traction by the introduction catheter. In the gastric fundus, the SGB was filled under direct visual examination with saline solution (mean of $650 \mathrm{ml}$ ), and fixed volumes of Iopamiron ${ }^{\circledR}$ contrast $(20 \mathrm{ml})$ and methylene blue to $2 \%(10 \mathrm{ml})$, in the final approximate proportion of $65: 2: 1$. The filling procedure was continually monitored so that a better adequacy of the SGB volume to the gastric capacity was achieved. After the filling procedure, the SGB was visually inspected for the detection of possible deflation and confirmation of the correct positioning in the gastric fundus (Fig. 3). Antiemetics and antispasmodics were administered orally or intravenously to control nausea and pain for 24 to $72 \mathrm{~h}$ when necessary. A proton pump inhibitor (PPI) of a dosage of 40 to $80 \mathrm{mg} /$ day was prescribed for all the patients during the treatment.

The first part of the SGB removal procedure was the positioning of a double silicon overtube in the patient's esophagus. Under direct visual observation, a hole was made by endo-scissors in each SGB, and a catheter inserted to empty the SGB. Alternatively, a specially developed catheter containing a needle was used to empty the SGB. Each completely emptied SGB was captured by a polipectomy snare and pulled until part of the SGB was held in the overtube, simultaneously allowing the removal of the whole endoscopic apparatus.

\section{Statistics}

To confirm the normal distribution of the efficiency variables of the 14 patients who completed the 6-month treatment, the Shapiro-Wilks test for normality was used. The $t$ test for paired observations under significance of 0.01 was used to evaluate the preliminary effectiveness of the proposed treatment. The descriptive statistics values are presented in the sections of "Methods" and "Results" as mean \pm standard deviation.

\section{Results}

In all cases, the SGBs were successfully placed and removed under usual sedation of diagnostic endoscopy.
Fig. 3 a Final positioning of the $\mathrm{SGB}$ in the gastric fundus. b The SGB in the final stage of the filling procedure
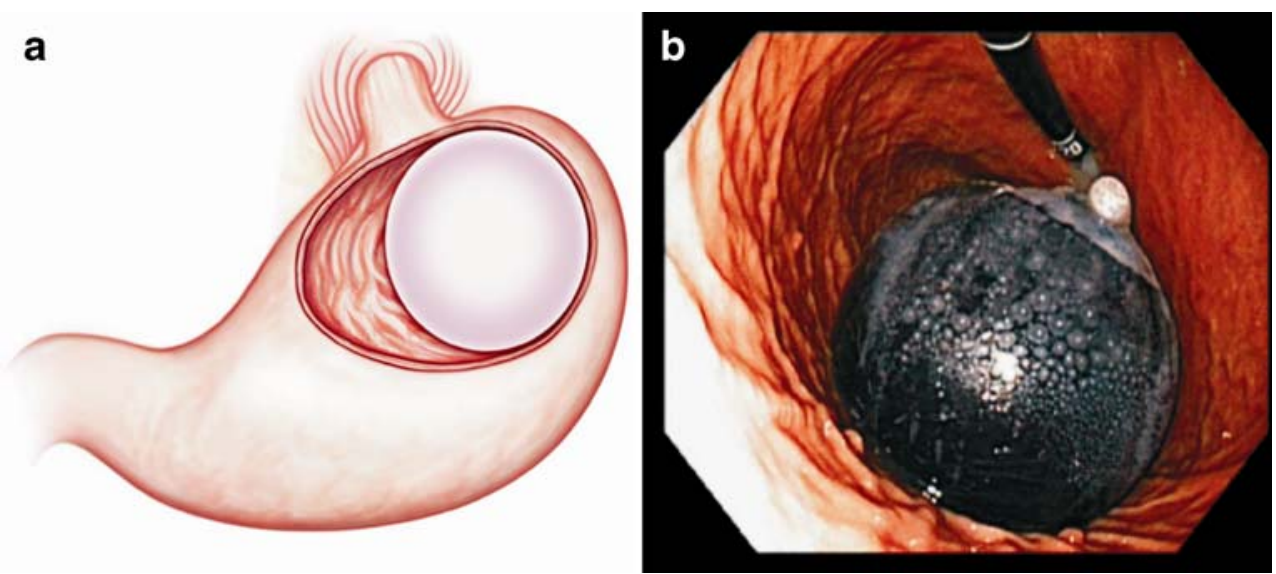
Table 1 Efficiency results of treatment with SGB in the 14 patients who had completed the 6-month treatment

\begin{tabular}{|c|c|c|c|c|c|c|}
\hline Patient no. & Filling solution (ml) & Initial Weight (kg) & Initial BMI $\left(\mathrm{kg} / \mathrm{m}^{2}\right)$ & Final BMI $\left(\mathrm{kg} / \mathrm{m}^{2}\right)$ & Weight Loss (kg) & EWL (\%) \\
\hline 1 & 650 & 80 & 30.5 & 27.0 & 9.0 & 62.5 \\
\hline 2 & 660 & 99 & 34.3 & 32.0 & 6.3 & 23.5 \\
\hline 3 & 580 & 100 & 38.1 & 35.2 & 7.5 & 21.8 \\
\hline 4 & 620 & 91 & 34.2 & 29.8 & 11.8 & 48.0 \\
\hline 5 & 630 & 85 & 32.0 & 27.8 & 11.0 & 59.2 \\
\hline 6 & 600 & 88 & 35.2 & 30.8 & 11.0 & 43.0 \\
\hline 7 & 700 & 155.1 & 44.8 & 38.4 & 22.1 & 32.2 \\
\hline 8 & 620 & 85 & 34.9 & 32.9 & 5.0 & 20.7 \\
\hline 9 & 650 & 86 & 32.6 & 30.5 & 5.6 & 27.7 \\
\hline 10 & 700 & 114.6 & 36.6 & 31.9 & 14.6 & 40.2 \\
\hline 11 & 650 & 85 & 30.8 & 25.7 & 14.0 & 87.0 \\
\hline 12 & 590 & 100 & 36.7 & 36.4 & 1.0 & 3.1 \\
\hline 13 & 690 & 157 & 50.1 & 43.7 & 20.0 & 25.4 \\
\hline 14 & 620 & 84 & 29.4 & 22.5 & 19.7 & 156.4 \\
\hline Mean & $640 \pm 38.6$ & $107.7 \pm 25.1$ & $35.7 \pm 5.7$ & $31.8 \pm 5.5$ & $11.3 \pm 6.2$ & $46.5 \pm 36.7$ \\
\hline Range & 580 to 700 & 80.0 to 157.0 & 29.4 to 50.1 & 22.5 to 43.7 & 1.0 to 22.1 & 3.1 to 156.4 \\
\hline
\end{tabular}

The procedures are simple and fast; the mean placement procedure time was $9 \mathrm{~min}(7-17 \mathrm{~min})$, and mean removal procedure time was $13 \mathrm{~min}(10-25 \mathrm{~min})$. The patients left the outpatient clinic in less than $1 \mathrm{~h}$ after the procedure. There was no intercurrence during the procedures and no instance of SGB loss in the esophagus or tracheal aspiration during the removal of the device. The use of Iopamiron ${ }^{\circledR}$ in the filling solution of SGB provided a better radiographic vision of the device during the treatment, when necessary.

All the 14 patients who had completed the 6-month treatment with SGB lost weight at the end of the treatment. Table 1 and Fig. 4 summarize the results of effectiveness. The $t$ test for paired observations under a significance of 0.01 showed that these preliminary results were significant.

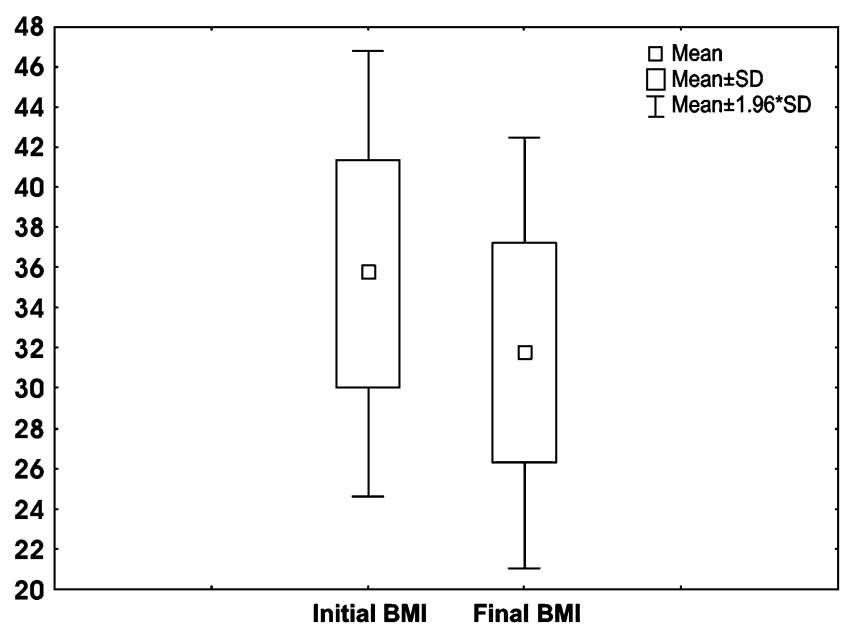

Fig. 4 Boxplot showing the means and dispersions of the initial and final BMI data of the 14 patients who had completed the 6-month treatment with SGB
The only initial complications were episodes of nausea, vomiting, and epigastric pain. Epigastric pain occurred in 11 patients $(21 \%)$, leading to early termination of the treatment.

There was no occurrence of late complications such as serious esophagitis, peptic ulcer and gastric perforation or erosion. The only late complications were two cases of spontaneous deflation of the device, one occurring after the 6-month treatment and the other within almost 6 months of treatment. In both cases, the deflated devices did not migrate to the intestine and were successfully removed by endoscopy, following the established protocol.

\section{Discussion}

The use of an intragastric device to induce the weight loss in obese patients was first described in 1982 [6]. However, the first gastric balloon designs did not yield the expected results of weight loss. On the contrary, they were associated with high incidence of complications, mainly as damage to the gastric mucosa and spontaneous deflation of the device, followed by intestinal obstruction in some cases [6-9]. This poor performance was due to technical aspects, such as the filling of the devices with air and the presence of a low resistant balloon shell with rough surface.

In 1999 was used a gastric balloon of silicon filled with saline solution and with a design that the manufacturer recommended its usage for 6 months [10]. Ever since, significant results have been obtained with this new balloon [1-4]. More recently, a prospective, double-blind, randomized, sham-controlled, and crossover study demonstrated that the procedure with the new device was more effective 
in treating obese patients than the sham procedure with restricted diet [3].

In the present study, based on the established concept of the intragastric balloon, the objective was to present an even safer and more effective treatment alternative for the weight loss in patients with appropriate indication. For this, the authors used a new intragastric balloon (SGB), and introduced technical improvements in the placement and removal procedures of the device.

In this series, all the SGBs were successfully placed and removed by endoscopy, with no intercurrences during the procedures. Both the procedures are simple and fast and, therefore, perfectly feasible under the usual sedation of diagnostic endoscopy, and under the ambulatory level in endoscopic suite, which avoids the risks and costs associated with general anesthesia and the surgical block.

Anchoring the extremity of the SGB's sheath to the extremity of the endoscope made the placement of the device by traction possible: placement by traction appeared to be simpler and more effective than placement by a tube that pushes the device without visual monitoring, as in the orogastric probe. In this stage, the direct visual monitoring enabled the fast positioning of the SGB in the gastric fundus, thereby reducing the excessive manipulation of the endoscope and the consequent risk of damage to the pharynx. The mean duration of the procedure-9 $\min (7$ to $17 \mathrm{~min}$ )—was shorter than the good results presented in some clinical series: Hervé et al. [11] and Genco et al. [3] obtained, respectively, a mean time of $14.5 \mathrm{~min}$ (10 to $30 \mathrm{~min}$ ) and $15 \mathrm{~min}$ (10 to $20 \mathrm{~min}$ ).

Although SGB has a radiopaque mark around the valve, the use of Iopamiron ${ }^{\circledR}$ in the filling solution of the SGB contributes to obtaining more clearly defined images on the correct placement of the balloon, whenever necessary. This contrast in the filling solution facilitates fine volumetric analysis of SGB, by overlapping of X-rays, in cases of suspected progressive deflation of the device. Moreover, according to the information supplied by the manufacturer of the device, the filling solution neither reacted with the SGB shell nor reduced the useful life of the device.

The stable catch of the SGB by the polipectomy snare, followed by the joining of SGB directly to the overtube, and the simultaneous removal of the whole endoscopic apparatus, constituted a very safe and effective removal procedure in all cases of 6-month treatment completion, and interrupted treatment due to intolerance to the device or balloon deflation. Even in the high pressure area of the esophagus, at the level of the cricopharyngeal muscle, the overtube containing the SGB passed through easily, thus minimizing the risk of damage to the esophagus or device loss in the digestive tract. The use of the overtube practically annulled the risk of tracheal aspiration of saline solution or food residues, thus making the procedure of tracheal intubation unnecessary for controlling these risks. Furthermore, the shortened removal time of SGB resulted in using fewer antispasmodics and decreasing thereby the patient's discomfort, mainly due to lower transparietal stimulation by the endoscopic apparatus and consequent reduction of the spasms of the cardia and the esophagus.

All the 14 patients who completed the 6-month treatment with SGB lost weight, the mean loss being $11.3 \pm 6.2 \mathrm{~kg}$, which is less than the losses of other larger clinical series: Hervé et al. [11] and Evans and Scott [1] obtained, respectively, a mean weight loss of $12 \mathrm{~kg}$ in 100 patients and $15 \mathrm{~kg}$ in 58 patients. The use of the $t$ test for paired observations showed that the preliminary results of weight loss were significant in this group of 14 patients, although the possibility of placebo effect or weight loss due only to behavioral changes can not be excluded. Nonetheless, two aspects that reinforce the effectiveness of SGB merit emphasis: (1) The recent publication of Genco et al. [3] showing that the intragastric balloon concept in the treatment of the weight loss in obese patients is more effective than the sham procedure associated with restricted diet. (2) The failure history of the patients of the present series to previous clinical treatments for weight loss.

In this series, there was no occurrence of any serious late complication such as erosion or peptic ulcer, except for two cases of late spontaneous deflation of the balloon. Even in these cases, the patients lost weight satisfactorily, and the devices were successfully removed by gastric endoscopy, thus reinforcing the concept of safety and effectiveness of the SGB treatment. The continuous use of the PPI during treatment is mandatory to ensure this safety by protecting the gastric mucosa and the balloon shell from the deleterious effect of the hydrochloric acid. As a result of this continuous use and consequent reduction in gastric acidity, the patient's digestive tract becomes a more favorable environment to the Candida $s p$, and this explains the colonization of the SGB shell by these fungi in some of the cases. In the symptomatic cases of candidiasis, during the SGB treatment, nistatin can be prescribed for the patient.

The treatment of weight loss using a new intragastric balloon presented encouraging preliminary results in terms of safety and effectiveness in eligible patients. Thus, the SGB can be considered a new reversible procedure for weight loss, which is minimally invasive and has low morbidity rate as compared to other bariatric procedures.

Open Access This article is distributed under the terms of the Creative Commons Attribution Noncommercial License which permits any noncommercial use, distribution, and reproduction in any medium, provided the original author(s) and source are credited. 


\section{References}

1. Evans JD, Scott MH. Intragastric balloon in the treatment of patients with morbid obesity. Br J Surg. 2001;88:1245-8.

2. Genco A, Bruni T, Doldi SB, et al. Bioenterics Intragastric Balloon: the Italian Experience with 2515 patients. Obes Surg. 2005; 15:1161-4.

3. Genco A, Cipriano M, Bacci V, et al. Bioenterics Intragastric Balloon $\left(\mathrm{BIB}^{\mathbb{R}}\right)$ : a short-term, double-blind, randomized, controlled, crossover study on weight reduction in morbidly obese patients. Int J Obes. 2006;30:129-33.

4. Sallet JA, Marchesini JB, Dyker SP, et al. Brazilian multicenter of the intragastric balloon. Obes Surg. 2004;14:991-8.

5. Schapiro M, Benjamin S, Blackburn G, et al. Obesity and the gastric balloon: a comprehensive workshop. Gastrointest Endosc. 1987;33:323-7.
6. Nieben OG, Harboe H. Intragastric balloon as an artificial bezoar for treatment of obesity. Lancet. 1982;1:198-9.

7. Durrans D, Taylor TV. The intragastric balloon, a new treatment for obesity. Clinical Nutrition. 1986;81:860-2.

8. Garren L. Garren gastric bubble. Bariatric Surgery. 1985;3: $14-5$.

9. Mathus-Vliegen EM, Tytgat GN, Veldhuyzen-Offermans EA. Intragastric balloon in the treatment of super-morbid obesity. Double-blind, sham-controlled, crossover evaluation of 500 milliliter balloon. Gastroenterology. 1990;85:833-7.

10. Bioenterics Intragastric Balloon $\left(\mathrm{BIB}^{\circledR}\right)$ System. Revisions to Indications for Use. Manufacturer's guidelines information sheet. Carpinteria, California: Bioenterics Corporation, 1999.

11. Herve J, Wahlen CH, Schaeken A, et al. What becomes of patients one year after the intragastric balloon has been removed? Obes Surg. 2005;15:864-70. 